\title{
Experimental Evolution in
} Plant-Microbe Systems: A Tool for Deciphering the Functioning and Evolution of Plant-Associated Microbial Communities

\section{OPEN ACCESS}

Edited by:

Ricardo S. Ramiro,

Gulbenkian Institute of Science (IGC),

Portugal

Reviewed by:

Israel Pagan,

Polytechnic University of Madrid

Spain

Inês Fragata

University of Lisbon, Portugal

Teppo Hiltunen,

University of Turku, Finland

*Correspondence:

Claire Prigent-Combaret claire.prigent-combaret@univ-lyon1.fr

Specialty section: This article was submitted to

Evolutionary and Genomic Microbiology,

a section of the journal

Frontiers in Microbiology

Received: 19 October 2020

Accepted: 29 March 2021

Published: 07 May 2021

Citation:

Manriquez $B$, Muller $D$ and

Prigent-Combaret C (2021)

Experimental Evolution

in Plant-Microbe Systems: A Tool

for Deciphering the Functioning and Evolution of Plant-Associated

Microbial Communities.

Front. Microbiol. 12:619122. doi: 10.3389/fmicb.2021.619122

\section{Beatriz Manriquez, Daniel Muller and Claire Prigent-Combaret*}

UMR 5557 Ecologie Microbienne, VetAgro Sup, CNRS, INRAE, University of Lyon, Université Claude Bernard Lyon 1, Villeurbanne, France

In natural environments, microbial communities must constantly adapt to stressful environmental conditions. The genetic and phenotypic mechanisms underlying the adaptive response of microbial communities to new (and often complex) environments can be tackled with a combination of experimental evolution and next generation sequencing. This combination allows to analyse the real-time evolution of microbial populations in response to imposed environmental factors or during the interaction with a host, by screening for phenotypic and genotypic changes over a multitude of identical experimental cycles. Experimental evolution (EE) coupled with comparative genomics has indeed facilitated the monitoring of bacterial genetic evolution and the understanding of adaptive evolution processes. Basically, EE studies had long been done on single strains, allowing to reveal the dynamics and genetic targets of natural selection and to uncover the correlation between genetic and phenotypic adaptive changes. However, species are always evolving in relation with other species and have to adapt not only to the environment itself but also to the biotic environment dynamically shaped by the other species. Nowadays, there is a growing interest to apply EE on microbial communities evolving under natural environments. In this paper, we provide a nonexhaustive review of microbial EE studies done with systems of increasing complexity (from single species, to synthetic communities and natural communities) and with a particular focus on studies between plants and plant-associated microorganisms. We highlight some of the mechanisms controlling the functioning of microbial species and their adaptive responses to environment changes and emphasize the importance of considering bacterial communities and complex environments in EE studies.

Keywords: experimental evolution, synthetic community, interaction network, microbiota, holobiont, evolutionary adaptation

\section{INTRODUCTION}

In nature, microorganisms are living inside complex microbial communities (i.e., microbiota) where they evolve under constant interaction with sympatric microbial populations (Brockhurst et al., 2003; Brockhurst and Koskella, 2013; Guan et al., 2013). While tremendous progress has been achieved regarding the description of natural microbial community composition, understanding 
their functioning, their structure dynamics and how they may evolve is still in its infancy (Rainey and Quistad, 2020). Myriads of interactions (e.g., cooperation, competition, or predation) occur between microbial community members which are influenced by all the biotic and abiotic factors the microbial community faced up. In turn, the functioning of microbial communities affects their environment leading to further changes in biotic interactions and in evolutionary dynamics of its members. Microbial communities are thus dynamical systems.

The environmental persistence and functioning of microbial communities are influenced not only by their species richness but also by their functional diversity. There are thus two important aspects of microbial communities: their taxonomic structure (diversity and abundance of each individual population within the community) and how they function (community behavior and activities) (Little et al., 2008). Microbial communities are composed of various functional groups which could be defined as all populations doing the same function (Bouffaud et al., 2016). The same population could belong to several functional groups, which leads to interconnected functional networks within a single microbial community (Bruto et al., 2014; Renoud et al., 2020). Reconstructing the structure of functional and metabolic networks within microbial communities and understanding how this structure might evolve over time is largely unknown even when imposing stable laboratory environmental conditions. However, there is a great expectation placed on experimental evolution (EE) studies to unravel the evolutionary dynamics of populations within microbial community, although EE studies on natural microbial community are so far uncommon.

Experimental evolution corresponds to the study of the evolutionary modifications occurring on populations in response to environmental conditions imposed by the experimenter (Kawecki et al., 2012). It made it possible to monitor the realtime adaptation of populations to their environment (biotic and abiotic) by observing evolution in real-time for ten, hundreds or even thousands of generations and detecting phenotypic or genetic changes between individuals in the populations. The use of experimental replicates allows to disentangle the contribution of chance events (such as drift and founder effects) and the contribution of the imposed selective pressure. Thus, unlike carrying out genomic analysis on existing natural organisms and then interpreting their evolution, EE makes it possible to transform evolutionary genetics into a prospective undertaking, and to decipher the genetic bases of adaptation (Van den Bergh et al., 2018).

The first experiments with continuous-cultures were employed in the early 1950s with microorganisms and focused on describing dominant mutant phenotypes favored during extended growth (reviewed in Adams and Rosenzweig, 2014). In the decades that followed, scientists attempted to correlate the observed phenotypic changes with gene mutations or duplications, revealing that genetic adaptation is the basis of evolutionary adaptation. Genetic changes happen rapidly, within the first hundred cell generations, for a bacterial monoculture grown in a unique and limited resource environment (Lenski and Travisano, 1994; Cooper and Lenski, 2000; Wiser et al., 2013; Kram et al., 2017; Lenski, 2017). Bacteria are powerful candidates for $\mathrm{EE}$ as they offer short generation time and large population size, so multiple mutations can be present simultaneously. Moreover, bacteria are easy to track (i.e., isolation, numeration) and store, this allows to compare competitive fitness between evolved and ancestral genotypes. A regular collection and conservation of samples during EE and further genomic, genetic and/or phenotypic analyses could be done to decipher the evolution process (Jerison and Desai, 2015; Marchetti et al., 2017). Indeed, genomic and molecular data are currently available for many bacterial species, as well as techniques for their precise genetic analysis and manipulation (Vacheron et al., 2018; Batstone et al., 2020; Li et al., 2020; Scheuerl et al., 2020; Rodríguez-Verdugo and Ackermann, 2021).

Nowadays, mass sequencing allows to unravel the genetic mechanisms underlying adaptation in bacteria at the genomic level (Kawecki et al., 2012; Bailey and Bataillon, 2016). EE can now be used to answer more complex ecological questions, such as evolutionary adaptation during biotic interactions between a bacterial population and a host or between bacterial populations within synthetic or natural microbial communities (Brockhurst and Koskella, 2013; Table 1 and Figure 1). It is also a powerful tool to decipher the underlying mechanisms of virus-bacteria coevolution (Paterson et al., 2010; Scanlan et al., 2011), adaptation of pathogens to humans (Yang et al., 2011) or evolution of animal gut microbiota (Lescat et al., 2017; Tso et al., 2018) but the latter topics are not discussed in the present review. Here, a particular focus was given, but not limited, to $\mathrm{EE}$ studies done with plant-microbe systems. We compare systems with increasing complexity (Table 1). First, using selected examples, we describe EE studies involving a single microbial species evolving under low complexity environmental conditions. These studies allowed to infer the genomic mechanisms underlying bacterial adaptation to new environments. Second, we analyse EE studies using more complex systems, such as those investigating the interaction between single microorganism and the plant. These EE studies shed light on the role of the eco-evolutionary feedbacks during microbe-plant interactions. Third, we examine the importance of considering synthetic or natural bacterial communities (or microbiota) and complex environments in EE studies. Finally, we discuss future avenues of EE studies and point out the gaps must be bridged to analyze complex systems with the same detailed analysis of genomics adaptation than simple systems (Figure 1).

\section{EXPERIMENTAL EVOLUTION TO DECIPHER THE ADAPTIVE EVOLUTION OF SINGLE MICROBIAL POPULATION IN SYNTHETIC SYSTEMS OF LOW COMPLEXITY}

Experimental evolution has mostly been carried out on very simple conditions: using a single organism evolving in a low complexity system (Barrick et al., 2009). These EE experiments have provided essential knowledge on the molecular evolution and adaptive changes of microorganisms, particularly bacteria, 
TABLE 1 | Selected examples of experimental evolution studies (with a focus on interactions with plants at the microcosm scale), that have contributed to advances in genomic evolution, horizontal gene transfer, and plant host-microbe interactions.

Simple synthetic media

Complex and/or fluctuating media

Microcosms with plant host

\begin{tabular}{cl} 
Wild-type & $\begin{array}{l}\text { Single strain evolution } \\
\text { - Genetic/genomic evolution }\end{array}$ \\
\hline 0 & - Transcriptional changes
\end{tabular}

- Transcriptional changes
- Adaptive radiation

- Escherichia coli single clone evolution (Lenski et al., - Adaptive radiation of Pseudomonas fluorescens

- Impact of the inoculum size (Garoff et al.. 2020) et al., 2013; Koza et al, 2017)

- Long-term evolution of E. coli (Kram et al., 2017)

- Importance of horizontal gene transfer in

Helicobacter pylori evolution (Woods et al.,

2020)

- Saccharomyces cerevisiae showing a transition to multicellularity life form (Ratcliff et al., 2012)

- Myxococcus xanthus evolution (Rendueles and

Velicer, 2017)
Mutant/variant Single strain evolution - Genetic/genomic evolution - Transcriptional changes - Adaptive radiation

\section{Several known species}

\section{co-evolution}

- Population dynamics

- Genetic/genomic evolution of each

species

- Biotic interactions evolution

- Gene transfer
- E. coli single clone evolutions (reviewed in Lenski, 2017)

- Bacterial-prey co-evolution (Nair et al., 2019; Scheuerl et al., 2019)

- Yeast mutualists co-evolution (Vidal and Segraves, 2020)

- Experimental co-evolution (Castledine et al., 2020)

- Impact of the diversity and composition of the synthetic community on the evolution of a given species (Osmond and de Mazancourt, 2013; Jousset et al., 2016; Calcagno et al., 2017)

- Neutral and selective dynamics in a synthetic microbial community (Cira et al., 2018)
- Adaptive radiation of $P$. fluorescens SBW25

$\triangle$ panB (Barrett et al., 2005)

- Myxococcus xanthus evolution (Rendueles and Velicer, 2020)

\section{- Fluctuating environment destabilizing bacteria} interactions (Rodríguez-Verdugo and Ackermann, 2021)

- Acinetobacter-Pseudomonas putida interaction evolution in a biofilm (Hansen et al., 2007)

- Impact on biotic interaction network (Lawrence et al., 2012; Cairns et al., 2018a)

- Importance of spatial structure (Cairns et al., 2018b) and toxic molecules (Piccardi et al., 2019)

- Community context affecting evolutionary

dynamics (Fiegna et al., 2015; Scheuerl et al. 2020)

Several species co-evolution
Population dynamics in the future:
single-cell genomic evolution and
horizontal gene transfer?

No studies in the literature but could allow, with meta-omics and single-cell sequencing, analysis of:

- Population dynamics

- Genetic mechanisms underlying species-specific adaptation within microbiota

- Genetic changes, at the cell level, within a community

Selection of adapted and stable microbiota applicable to agriculture or medicine issues
- Ralstonia solanacearum pathogen adaptation to plants (Guidot et al 2014; Gopalan-Nair et al., 2020)

- Pseudomonas protegens adaptation to Arabidospis ( $L i$ et al., 2020

- Modified Ralstonia solanacearum evolving in a plant symbiont (Marchetti et al., 2010; Doin de Moura et al., 2020)

\section{- Pseudomonas syringae} adaptation on its natural host or on a distant one in the presence or absence of bacteriophages (Meaden and Koskella, 2017)

- Enhanced cooperation with different plant genotypes of Ensifer meliloti in competition with a nitrogen-fixing defective cheate (Batstone et al., 2020)

- Adaptation of microbiota to host plants (Morella et al., 2020)

- Evolution of rhizosphere microbiota to influence plant growth parameters (flowering, biomass) (Swenson et al., 2000; Panke-Buisse et al., 2015; Lu et al., 2018) 
A

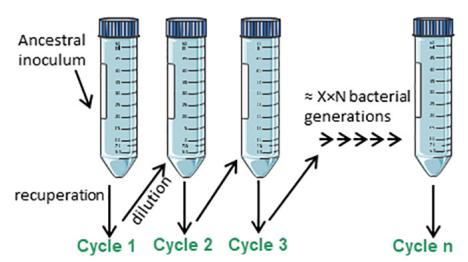

Main outcome:

- Adaptation

- Radiation

Measures of phenotypic traits

Genetic and genomic evolution

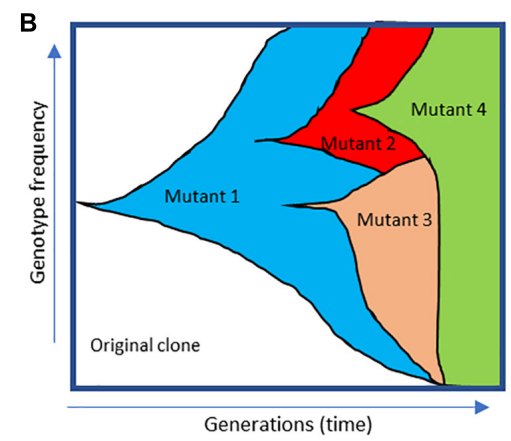

C

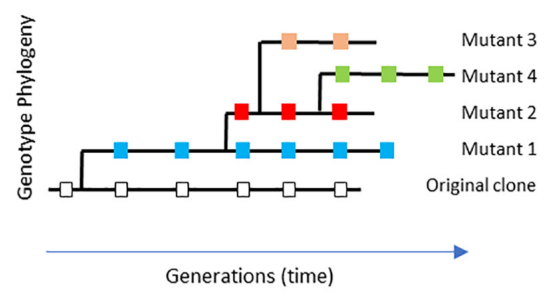

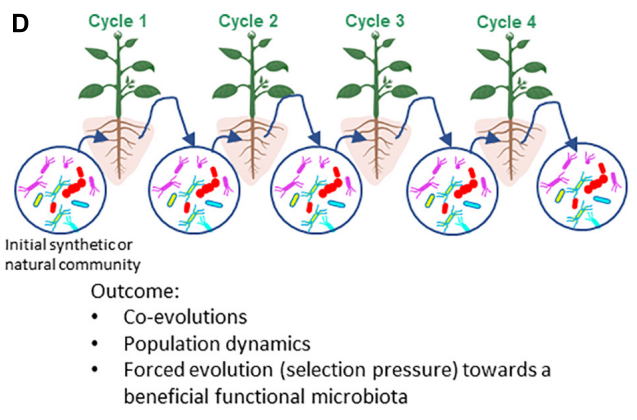

E 100

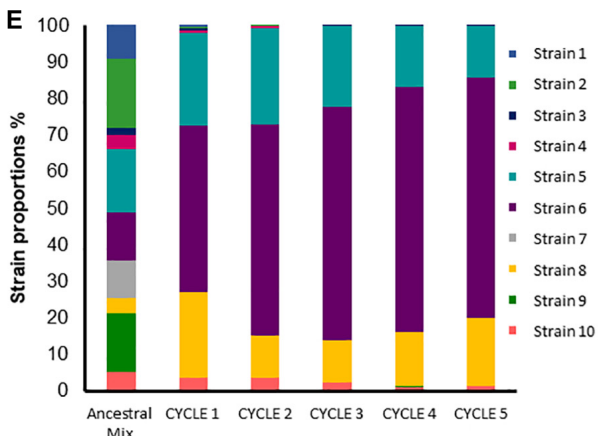

$\mathbf{F}$

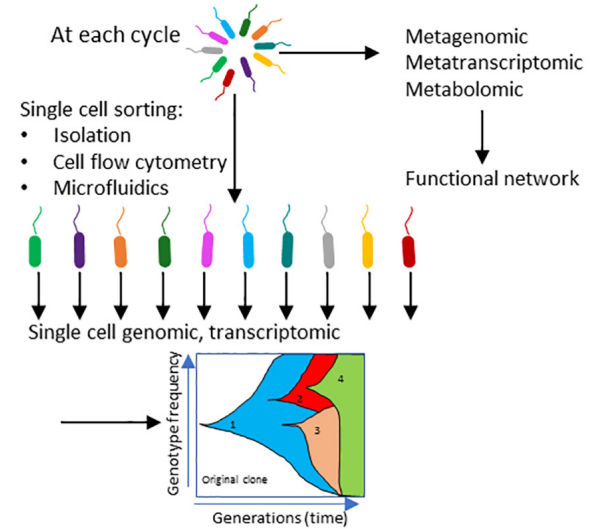

FIGURE 1 | Comparison of the potential outcomes from experimental evolution (EE) studies starting with (A) a single clone or (D) a microbial community. (A) Example of an experimental evolutionary design based on a single clone serially cultivated in a simple medium, a stressful or a changing environment; those experiments enable to track phenotypic and genetic changes over time. (B) Theoretical Muller diagram depicting the distinct genotypes frequencies over microbial generations that could be observed in EE study described in A. (C) Theoretical phylogenetic tree built from genotypes that can be sampled from different time points throughout the EE described in A and sampled at the same generation times. (D) Example of an experimental evolutionary design based on an inoculum made up of several microorganisms (synthetic or natural communities); those experiments make it possible to monitor changes in population levels (E), and genetic, transcriptomic or metabolomic changes over time (F). (E) Theoretical bar graph illustrating the composition of a microbial community showing differences in taxa among both the inoculum (ancestral mix) and different experimental cycles. (F) At each cycle, approaches could be implemented at the scale of the whole community (i.e., meta-omics) or of individual cells after cell-sorting (single-cell sequencing, transcriptomic).

to experimenter-imposed conditions and allowed to uncover the correlation between genetic and phenotypic changes (Figure 1).

\section{Bacterial Adaption and Key Evolutionary Driving Forces}

Adaptation of populations mostly happens through mutations that can improve the performance of organisms in their environment and therefore improve their fitness
(Lang et al., 2011). Distinct environmental conditions will impose different selection pressures and will determine whether or not a mutation would be beneficial or detrimental in a given niche at a given time. If a beneficial mutation happens on a single clone it will need a certain amount of time to rise to the population level (fixation). According to Fisher's model, the time required for a mutation to fix is inversely proportional to its beneficial effect (Fisher, 1930; Tenaillon, 2014). However, stochastic extinction of a mutation, even if it 
may confer some fitness advantages, or increase in frequency of a deleterious mutation can exist. So called "genetic drift" also plays an important role in fixation of mutation in a population and represents the role of chance in evolution (Kayser et al., 2018; McDonald, 2019). The spatial distribution of cells will also influence the genetic drift's force and competition between mutated clones (Kayser et al., 2018). However, bacteria reproduce asexually, so the different genotypes that rise in the population do not recombine and the corresponding mutations they harbor may not reach fixation within the population. This phenomenon is known as clonal interference and influences the dynamics of evolution and adaptation as clones with beneficial mutations will interfere with each other's spread in the population (Gerrish and Lenski, 1998; McDonald, 2019). However, motility and dispersal may help competitive populations to coexist in a same system through active segregation and spatial exclusion (Gude et al., 2020).

\section{Parallel and Convergent Evolution of Single Microbial Species in Simple Environments}

Given that evolution in bacteria happens mostly by random mutations, an important question arises from EE studies: are they repeatable? Do same genotypic and phenotypic changes arise in an evolved microbial population submitted to same environmental changes? In order to better understand evolutionary adaptation, genotype-to-phenotype correlations need to be more fully investigated. This can be done by focusing on patterns of parallel and convergent evolution.

Parallel evolution is defined as the independent evolution of similar phenotypic traits in lineages closely related to each other and involving changes in orthologous genes, whereas convergent evolution concerns non-related phylogenetic lineages and changes in non-homologous genes (Bai et al., 2015; Pickersgill, 2018).

Many EE studies have shown that a single clonal ancestor can give rise to independently evolved populations sharing similar traits under the same environmental conditions (Schluter et al., 2004; Colosimo, 2005; Zhang et al., 2005). Lenski's experimental evolution study is composed of 12 replicate populations of E. coli B, which are currently still evolving on a glucoselimited minimal salts medium (Lenski et al., 1991; Lenski, 2017; Consuegra et al., 2021; Table 1). DNA microarray analyses of two evolved populations showed similar changes in the transcription of 59 genes after 20,000 generations; the genetic bases of these changes were investigated and relevant parallel mutations were found on many of the independently evolved populations (Cooper et al., 2003).

In simple organisms, biological functions are mostly encoded by single genes, whereas, in higher forms of life, more complex regulatory networks are involved. In bacteria and yeast, complex functions are often related to modules of genes (Hartwell et al., 1999). Reflecting this, parallel evolution will not always mean changes in the same genes but rather similar changes in related gene modules. Herring et al. (2006) explored the parallel changes in metabolic and regulatory networks that arose in five E. coli populations that evolved separately. They proved, combining mutant approach and whole-genome resequencing of five clones of $E$. coli, that 13 different spontaneous mutations were responsible for improved fitness during adaptation to a glycerol synthetic growth medium (Herring et al., 2006). This study provides a clear example of how different changes can have similar phenotypic effects (Table 1). Dissimilar genetic changes can lead to parallel phenotypes, meaning that functional connections within and between genetic modules can be established by pairing experimental evolution to whole genome sequencing (Segrè et al., 2006; Yeh et al., 2006).

However, clonal populations evolving in an identical environment could reach different fitness rates and may diverge (Lenski et al., 1991). This divergence reveals that there may be more than one adaptive strategies under the same environmental conditions (Elena and Lenski, 2003). On the other hand, we could expect that experiments ran with genetically different organisms led to different fitness. Travisano et al. (1995) evaluated fitness on maltose of several E. coli lines that had evolved in glucose for 2,000 generations. Lines that started with the lower fitness in maltose improved faster, but all the lines tend eventually to converge to a similar fitness on maltose evidencing convergent evolution.

\section{EE of Single Microbial Species in Variable and Complex Environments}

In the past, EE studies were mostly done in simple environments, where the concentration of one essential source of carbon available to all individuals controls the population growth rate (Hillesland and Stahl, 2010; Yu et al., 2017).

This selects for different types of genotypes throughout experiment transfers. Heavy consumers and fast-growing microbes show increased fitness in the beginning of the experiment. When the resource starts to be depleted, genotypes that are able to survive with low resources and metabolic by-products increase in frequency. Finally, when there are almost no resources, there is an increase in prevalence of genotypes that are able to either metabolize toxic by-products (e.g., Blount et al., 2012) or are able to grow in very small amount of resources. This may lead to the evolution of two types of genotypes: generalist (able to consume a large range of the available resources) or specialist (able to grow faster than generalists, but in a shorter range of resources). This ensures the maintenance of polymorphism in population, with all genotypes present in the population, but with their prevalence varying until the cycle is started again with a new medium transfer. In complex environments that contain several resources, cell populations may become wide specialists able to consume various carbon substrates.

In the laboratory, because of the complexity of evolutionary processes, most of the EE assays have been carried out in a constant environment, using chemostats or continuous culture systems, so the growth conditions would remain the same for the whole experiment (Treves et al., 1998; Maharjan et al., 2006; Gresham and Hong, 2015). Recently, Westphal et al. (2018) used a batch culture system to study the impact of four changing environments on the adaptation of $E$. coli. In these systems there is no addition of nutrients, therefore the cultures experience fluctuation in nutrient availability. Therefore, as nutrients are consumed, waste products are released and 
less energetically favorable metabolisms become important for survival. Their data revealed that different environments may select different mutations; this emphasizes the importance of performing experimental evolution in complex and everchanging environments. To take these findings one step further, Kram et al. (2017) carried out an EE assay with E. coli in a complex and variable environment (i.e., in a rich medium LB and with serial passages every 4 days). This scheme allows cells to go through all phases of growth and to adapt to different stresses (nutrient limitation, oxidative stress and $\mathrm{pH}$ variation). This experiment showed that after only 30 generations, evolved populations presented changes in growth rates but also adaptive mutations allowing the cells to cope with the varying stresses arising during the culture (Table 1). They also evidenced parallel changes in evolved populations (Kram et al., 2017).

The importance of environmental complexity and evolution of niche width have been studied by Barrett et al. (2005) who compared the evolution of more than one hundred replicate lines of the bacterium Pseudomonas fluorescens, over $\sim 900$ generations in 15 environments of different complexity (Table 1). To do this, the authors used a synthetic growth medium that contained 1-8 single carbon substrates or specific combinations between them, and compared the genetic evolution of $P$. fluorescens lineages in simple and complex environmental growth media. In complex medium, the Pseudomonas lines evolved into several co-existing genotypes (adaptive radiation), exhibiting greater fitness for a wider range of carbon sources (but not for all), than the lines that evolved in simple environments. A higher fitness variance within populations selected in complex environments was thus observed. Indeed, lineages evolved in simple environment specialized in consuming a single carbon substrate, while those evolved in complex media were able to consume several substrates (but not all), without any appreciable loss of functions or apparent fitness costs. These results suggest that evolution in complex environments will lead to the emergence of imperfect generalist overlapping lines, adapted to a certain range of substrates but not to all (Barrett et al., 2005).

In nature, communities grow under conditions where many substrates are available, supporting a great number of consumer strategies for microorganisms. However, the availability of these substrates can be heterogeneous (both in space and time), leading to fluctuating selection for different genotypes within microbial populations, or to varying species within the microbial community. The presence of biotic interactions can also be a cause of increased environmental complexity (Brockhurst and Koskella, 2013). However, this environmental component was largely overlooked until more recently. A great example of how biotic interactions provide spatial and temporal heterogeneity is the plant rhizosphere (Kuzyakov and Razavi, 2019; Figure 2 and Box 1).

\section{EXPERIMENTAL EVOLUTION WITH COMPLEX PLANT-MICROBE SYSTEMS}

Plant select microorganisms in the rhizosphere through their exudates (Figure 2). Interactions between plant and microorganisms can be beneficial, neutral or deleterious and several studies have used EE to unravel the relationships between the plant and plant-associated microorganisms (Table 1). Here we review how these EE assays have helped to better understand plant-microorganism's interactions, first for plant pathogens and secondly for plant-beneficial bacteria.

\section{Plant Pathogens}

Plant pathogens are pervasive, and their management is important for agriculture and food supplies, with direct impacts in human health and welfare. Thus, understanding the mechanisms underlying host co-evolution can help on devising new strategies to eradicate these types of pathogens. It is known that pathogen and plant defenses co-evolve, which translates into an arms race, where host and pathogen constantly evolve by mirroring the response of one another (Brockhurst and Koskella, 2013). Deciphering the genetic bases of pathogen adaptation is thus critical to understand disease emergence and acquisition of novel traits by pathogens when colonizing hosts (Toft and Andersson, 2010; Gandon et al., 2013).

EE approaches have been widely used to study the ecology of plant pathogens and dynamics of their adaptation when they interact with new host. Here, a focus is made on in planta EE experiments done with bacterial plant pathogens (Table 1), but others studies have also focused on fungal (Gilbert and Parker, 2010) or virus plant pathogens (Bedhomme et al., 2012).

Indeed, in an attempt to experimentally study the genetic basis of adaptation to new host, Guidot et al. (2014) performed EE with Ralstonia solanacearum, a plant pathogen with a continuously broadening host spectrum. A single clone of the model strain GMI1000 was inoculated on three native host plants (tomato, eggplant and pelargonium) where the pathogen causes disease and two distant plants (cabbage and bean) where it grows asymptomatically. The pathogen was transferred serially to the same plant line, in order to maintain the pathogen on the same host for 300 bacterial generations (26 serial passages). Although evolved strains showed an increase in competitiveness (pathogenicity) in both host and non-host plants, this increase was greater in the non-host plant (cabbage and beans), to which the pathogen was originally not adapted. This rapid evolution when colonizing a distant host plant tends then to decrease until reaching an optimum. This is known as "diminishing returns epistasis" that is often observed during EE studies with almost invariantly a reduction of adaptation speed and of mutations' fitness gain during the adaptation (Couce and Tenaillon, 2015).

Whole-genome analysis and comparison of the ancestral GMI1000 strain with nine evolved clones (three from the tomato host and six from the bean) highlighted that only few genes contribute to adaptation to a specific host. In particular, the transcriptional regulator encoding gene efpR was identified as important for the adaptation of $R$. solanacearum to bean. Evolved clones harboring efpR mutations had a greater competitiveness compared to the wild type clone during co-infection of bean plants (Guidot et al., 2014). EfpR was thereafter identified as a global catabolic repressor and regulator of virulence traits, whose 

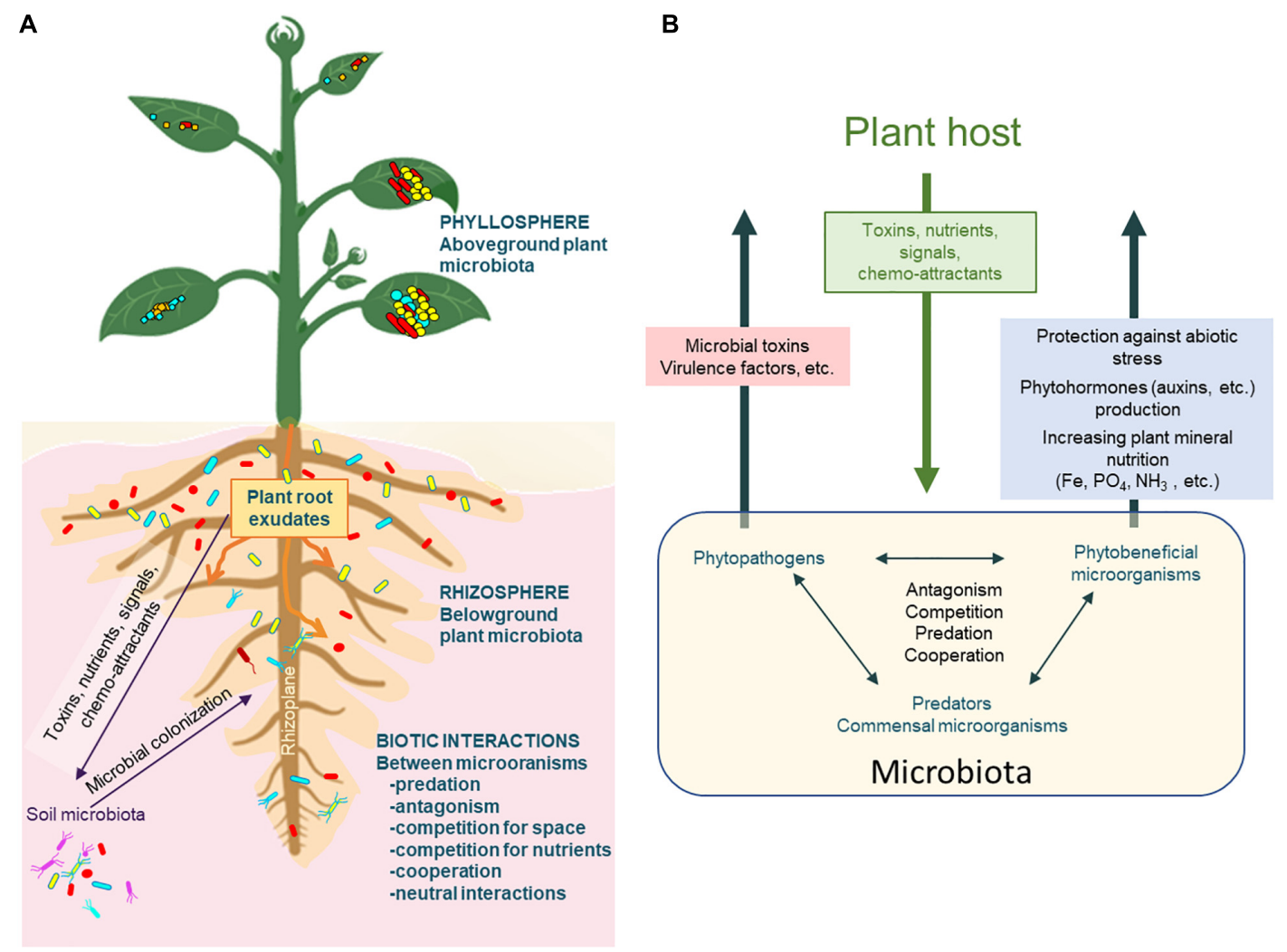

FIGURE 2 | Relationships between plant and microbial components. (A) Plants interact with a wide diversity of microorganisms (microbiota) in the different compartments of the plant system. The rhizosphere corresponds to the soil zone where the roots impact the soil organization and microbial functioning, while the rhizoplane is the interface between the root surface and the soil. The phyllosphere corresponds to the surface of all the aerial organs of the plant. Root exudates attract or repel soil microorganisms toward roots and increase the growth of myriads of microorganisms that will interact between each other (positive, negative or neutral biotic interactions). (B) Major biotic interactions in the rhizosphere include plant-microorganism interactions and microorganism/microorganism interactions, involving the exchange of different classes of molecules (hormones, toxins, virulence factors, signals, etc.).

BOX 1 | The plant rhizosphere, a rich and heterogeneous environment. Many soil microorganisms are capable of colonizing the plant rhizosphere, the volume of soil under the influence of root activities. In the rhizosphere plants exert a selection on soil microorganisms by secreting around their roots a wide range of compounds known as plant exudates (Figure 2). About 5-21\% of the total carbon fixed by photosynthesis has been reported to be exuded at the root level and to influence the composition of the rhizosphere microbiota (Derrien et al., 2004; Vandenkoornhuyse et al., 2015). The exudates contain, among other things, nutrients but also signaling and chemo-attracting molecules. Along the root system, the exuded molecules are not evenly distributed. This leads to a heterogeneous root and rhizoplane colonization by selected microorganisms able to use the nutrients locally available. Some of these microorganisms will cooperate with the host plant through diverse mechanisms, like increasing the plant's mineral and nitrogen nutrition, synthesizing phytohormones influencing plant phenotypes or stimulating the induced systemic response (Vacheron et al., 2013; Figure 2). The nature of the exudates will impact the composition and functions of the root-associated microbiota which in turn will affect the physiology of the plant, generating new exudates (Vacheron et al., 2013; Chaparro et al., 2014). Thus, the rhizosphere is a discontinuous environment both in space and time, rich in nutrients, locally increasing the growth of highly diverse microbial populations that are ultimately in constant competition for nutrients. These competitive conditions make the rhizosphere a hotspot for horizontal gene transfer (HGT, van Elsas et al., 2003), mutations or gene arrangements, and will drive bacterial adaptation and acquisition of new genes (Wisniewski-Dyé et al., 2011). The latter will result in population genotype and phenotype changes, in the modification of the structure and function of the community and hence, will have profound effects on the long-term evolution of communities. Nevertheless, understanding how rhizosphere community members may evolve, at a species, sub-species or even individual cell level, is still a complex issue. Right now, rhizosphere natural communities and plant rhizosphere systems have rarely been included in EE studies. But, thanks to the development of single cells sequencing, considering such complex and heterogeneous living system in EE studies is becoming feasible and will allow to obtain a comprehensive detailed view of rhizosphere communities functioning and driving forces (Figure 1).

mutations allow an enhanced metabolic versatility and adaptation to host vascular tissues of new hosts (Perrier et al., 2016). This study highlighted the importance of combining $\mathrm{EE}$ with whole-genome sequencing in order to unravel the genetic basis of pathogen adaptation. More recently, transcriptomic analyses combined with genomic sequencing evidenced that epigenetic modifications also occur during the adaptive evolution of $R$. solanacearum to a tomato resistant cultivar, allowing expression changes of the EfpR regulon (Gopalan-Nair et al., 2020).

Another EE study focused on understanding how the prior evolutionary history of a pathogen affected subsequent evolution 
in a new host. To this end, Pseudomonas syringae, a wellknown plant pathogen, has been used to infect a distant host (Arabidopsis) or a native host (tomato) over several infection cycles, in the presence or absence of phages. Bacteriophages impose evolutionary trade-offs on the bacteria they infect, triggering modifications of bacterial surface receptors that subsequently may impact $P$. syringae interactions with distinct hosts. Selection on Arabidopsis (through serial passaging) leads to a larger increase in pathogen growth rate on both hosts, than selection on tomato. These results point out that a given association between a plant and a pathogen may affect the growth rate of the pathogen on other plants it will subsequently infect (Meaden and Koskella, 2017).

Other EE studies done with Agrobacterium have highlighted the appearance of cheaters in pathogen populations that will benefit of the production of virulence factors by the rest of the population without producing them themselves (Tannières et al., 2017). Indeed, the production and regulation of virulence factors are usually associated with high costs (energy or fitness) and are finely regulated by quorum sensing signals in Agrobacterium. Tannières et al. (2017) showed that cheater mutants that minimize the costs of expressing quorum sensing regulated functions spread during EE.

\section{Non-pathogenic Plant Associated Microorganisms}

EE studies have also investigated the genetic mechanisms underlying adaptation of bacteria that present mutualistic or synergistic plant-microbe interactions.

Fabaceae-rhizobium interactions are known as highly specific interactions between a bacterial symbiont and its host plant. In this case, the plant rewards cooperative symbionts in detriment of less mutualistic microbes but without necessarily punishing the latter (Batstone et al., 2017). Using a year-long EE experiment between Ensifer meliloti and Medicago truncatula, Batstone et al. (2020) have shown that local and recent adaptation of the symbiont to a plant genotype increases cooperation, independently of host selection.

Another EE experiment has investigated which bacterial genes facilitate symbiosis between bacteria and plant using a legume symbiont and a non-symbiotic bacterium. To do this, $R$. solanacearum, hosting the Cupriavidus taiwanensis symbiotic plasmid of Mimosa pudica, was repeatedly inoculated on the plant host (Marchetti et al., 2010; Table 1). After a series of Mimosa pudica infection cycles, an evolved symbiont well adapted to the host (i.e., able to induce nodulation and infect nodules, however, not to fix nitrogen) was obtained. Sequencing of intermediate and final forms revealed that the symbiosis-adaptive mutations happened in global regulatory proteins, leading to a reworking of the regulatory systems in $R$. solanacearum. These adaptive mutations included the inactivation of the type III secretion systems (the main virulence factor of $R$. solanacearum; Genin and Denny, 2012) and modifications on the expression of $e f p R$ (Guan et al., 2013; Capela et al., 2017). Genomic analyses revealed that is not only the efpR gene that is mutated but also its upstream region. Altogether these mutations led to metabolic and transcriptomic changes, allowing mutualistic interaction with the plant (Capela et al., 2017; Marchetti et al., 2017; Doin de Moura et al., 2020). These findings highlight how EE can help to identify the evolutionary pathways that drive the evolution of symbiotic functions in rhizobia, by understanding how adaptation modifies the regulatory systems that control virulence and determine the ecological functions of bacteria.

Other plant-beneficial bacteria are involved in less specific interactions with plant. These bacteria known as Plant GrowthPromoting Rhizobacteria (PGPR) colonize the plant rhizosphere (Figure 2). EE assays carried out in planta with plant-inoculated PGPR are uncommon. To our knowledge there is only one open resource work dealing with EE during plant-PGPR interaction (Li et al., 2020). In this work, a well-known biocontrol PGPR Pseudomonas protegens CHA0 was grown on the roots of Arabidopsis thaliana (on five independent Col-0 replicates) in gnotobiotic conditions, during 6 months (i.e., six one-month plant growth cycles). The bacterium was shown to evolve to a more mutualistic relationships with its plant, acquiring an improved competitiveness for root exudates, a better ability to tolerate plant-secreted antimicrobial compounds and a stronger positive effect on the plant performance. Different mutations in the key two-component regulator system GacS/GacA were recorded, conferring higher competitiveness to evolved CHA0 clones compared to the ancestral form in the presence of A. thaliana plants.

\section{EXPERIMENTAL EVOLUTION STUDIES WITH SYNTHETIC AND NATURAL MICROBIAL COMMUNITIES}

A large portion of studies on EE and microbial adaptation focuses on one single species, considering the genetic diversity within an evolving population rather than diversity between species within communities (e.g., Wiser et al., 2013; Kram et al., 2017; Lenski, 2017; Garoff et al., 2020). However, microorganisms are indeed in constant interaction with other organisms in their environment, so that any population in nature evolves with the other sympatric microbial populations.

Species interactions can influence how species evolve and adapt to environmental changes (Gorter et al., 2020). Unraveling the interactions that take place within a community is essential to understand how a community carries a function and how it will respond to perturbations and may evolve. For example, within a microbial community, some species can use the waste products generated by others (Lawrence et al., 2012; Seth and Taga, 2014; Piccardi et al., 2019). These interactions can become so relevant that a given microorganism can present a lower growth rate or even may not grow when cultivated alone, compared to when cultivated with another microorganism (Hillesland and Stahl, 2010) or within a community (Smith and Schuster, 2019). However, adaptation of one population to a new environment could be favored or counteracted by sympatric populations (Castledine et al., 2020). This process of reciprocal adaptation by interacting species is defined as co-evolution (Buckling and Rainey, 2002). Sympatric populations could be 
locally maladapted and may show trait convergence for the use of same resource and these competitive interactions are specific to the co-evolved community members (Castledine et al., 2020).

\section{EE With Synthetic Community in Environments of Increasing Complexity}

Evolving together creates adaptive co-evolutionary dependencies: "it takes all the running you can do to stay in the same place," meaning that species have to constantly adapt to the other evolving species in order to survive/maintain their fitness (i.e., "Red Queen hypothesis"; Van Valen, 1977; Strotz et al., 2018). Species that co-evolved increased competitive interactions between them. Coevolution between competitors is expected to change species abundances within the community and affect subsequent community evolution (Nair et al., 2019; Scheuerl et al., 2019, 2020; Figure 1).

Thus, co-evolution experiments focused initially on microorganisms sharing negative interactions (i.e., predation, antagonism) (Nair et al., 2019; Scheuerl et al., 2019). Nowadays, co-evolution experiments also focus on mutualistic interactions, because these interactions are widespread in nature (Chomicki et al., 2020).

Several EE studies investigate the evolution of pairwise interactions between organisms (Nair et al., 2019; Scheuerl et al., 2019; Vidal and Segraves, 2020; Rodríguez-Verdugo and Ackermann, 2021). Here again, EE can be performed in constant environment (controlled microcosms with stable nutritive and physicochemical parameters), or in a fluctuating or complex environment (Table 1). Changes in the environment strongly constrain the existing biotic interactions between microorganisms. Rodríguez-Verdugo and Ackermann (2021) compared the evolution of co-cultures of Acinetobacter johnsonii and Pseudomonas putida in constant environment (the same nutrient medium at each cycle) to EE conducted in fluctuating environment (alternation of nutrient sources between each cycle). They showed that, the two species coexisted over 200 generations in the constant environment, whereas in the fluctuating environment, the extinction of one of the two partners was observed in half of the repetition, suggesting that the fluctuating environment destabilizes positive pairwise interactions.

However, natural microbiota are generally more complex and host a multitude of species of microorganisms, that will directly impact the evolutionary trajectory of each population (Jousset et al., 2016; Cira et al., 2018; Hall et al., 2020; Scheuerl et al., 2020), for example by suppressing competitors (Osmond and de Mazancourt, 2013), or by generating new niches (Calcagno et al., 2017). Species have thus to adapt not only to the environment itself but also to the biotic environment dynamically shaped by the other species (Ratzke and Gore, 2018; Scheuerl et al., 2020). Sharing the same ecological niche implies sharing some resources and this inevitably promotes competition within the group. Synthetic community approaches aim to mimic natural microbiota (Vorholt et al., 2017) and can help to better understand the functioning of biotic interaction network within natural communities (Figure 1). Cairns et al. (2018a) serially transferred a synthetic community of 33 bacterial strains on a complex liquid media. Over half of the strains from different species were lost in 16 days, after which the evolved community was relatively undisturbed until the end of the experiment (48 days). Within the evolved community, 14 strains co-existed with the predominance of three strains. The evolved synthetic community shared high diversity at different levels (i.e., taxonomic, metabolic, and functional levels) (Table 1).

Adaption to other competitive species may imply the production of antimicrobial metabolites, but a trade-off can arise due to the substantial energy-cost of their production (Yan et al., 2018). Some compounds can be costly to produce for one individual, but beneficial for all the members of the community. Microbes have thus developed multicellular cooperative behaviors, like biofilm formation and quorum sensing, along with nutrition acquisition, and the outcome of these interactions are referred as public goods (Besset-Manzoni et al., 2018; Smith and Schuster, 2019). Public goods take many forms from large proteins to small metabolites and can be actively or passively secreted, but one of their main features is that their benefit increases with population density.

Various EE studies comparing synthetic microbiota whose complexity increases (diversity and/or richness), increasing also the complexity of biotic interactions (competition for resources, cooperation, etc.), show that species adaptation largely depends on the community in which these species co-evolve. Thus, factors such as diversity or richness must be considered in microbial adaptation and evolution (Fiegna et al., 2015; Scheuerl et al., 2020). Another factor to consider in microbiota evolution is the impact of harsh conditions which may stimulate competition or cooperation. In a $\mathrm{EE}$ performed with synthetic microbiota, Scheuerl et al. (2020) adapted a continuous culture protocol and replaced the fresh medium addition at the end of each evolutionary cycle with the addition of only $10 \%$ of fresh medium, mimicking more natural conditions, and inducing competition and adaptation to recalcitrant carbon sources. This EE study revealed that the adaptation of bacteria to new environment is influenced by interspecies interactions.

Experimental evolution studies also enable to investigate the outcomes of interspecific interactions. For example, it is expected that plant root microbiota will be subjected to large environmental changes during plant development, which may lead to subsequent adaptation of the microbial community (Box 1). Specifically, a large number of natural compounds are exuded in the soil surrounding the roots, especially near the young parts, which modifies the physio-chemistry of the soil (oxygenation, $\mathrm{pH}$, etc.), generating a stressful environment for the microbiota. Such stressful environment can result in competition or cooperation between members of the community. Using a synthetic community composed of four bacterial species, Piccardi et al. (2019) showed that the interactions between species evolved differently if the environmental stress level is low or high (stress gradient). Indeed, in a mild-stress environment, species evolved competitive interactions whereas, in harsh and toxic conditions, members of the community evolved cooperation or neutral behavior. 
Unfortunately, cooperative behavior is not the only evolutionarily stable strategy as cheaters may appear and invade the community. Cheaters are non-cooperative individuals that benefit from the public goods without producing them. By not paying the cost of their production, cheaters could have more energy allocated to their growth, and therefore their relative fitness increases. The underlying mechanisms of cheaters' loss of function often involve a selective gene loss to optimize their adaptation to the environment (i.e., the "Black Queen Hypothesis," Mas et al., 2016). This evolutionary strategy has great impact on long term interactions because the fitness of cheaters depends on the public goods provided by the cooperating microbes. The stability of the community can only be maintained if the proportion of cheaters remains low within the community. Ultimately, cheaters will reduce the effective population size of the cooperating microbes, reducing the rate of public goods production, but also the rate at which beneficial mutations would arise in the community and the species would be able to adapt to a novel environment. The evolution of cooperation is a tricky issue since all microorganisms will tend, according to the natural selection theory, to maximize their own fitness. However, cooperative behavior is ubiquitous even though selfish interests have always been a source of conflict (Sachs and Wilcox, 2006; Burt and Trivers, 2009; Oliveira et al., 2014).

Batstone et al. (2020) investigated the prevalence of cheaters and non-cheaters in a simplified plant root community by performing EE with two strains of Ensifer meliloti. Both of these strains were able to receive carbon from the plant, but one strain lost the ability to fix $\mathrm{N}_{2}$ to feed back to the plant (cheater), and another strain maintained that function (cooperative). After 1 year, the authors observed that the frequency of these two strains varied according to the level of coevolution with the plant. On one hand, in the initial stages of the EE, the cheater presented a twofold fitness advantage in host colonization, but by the end of the experiment it was extinct in the five plant genotypes tested. On the other hand, the $\mathrm{N}_{2}$-fixing bacteria, which are less efficient in the first cycles of EE, became dominant at the end of EE. The interaction of evolved clones with the five Medicago lines showed that evolved clones achieved a higher fitness and provided greater benefits on the genotype with which they shared evolutionary history. Overall, this study suggests that cheaters are not able to outcompete cooperative genotypes, once they co-evolved with their host (Batstone et al., 2020).

Despite these recent studies, we still lack information on the eco-evolutionary dynamics behind cooperative and mutualistic behaviors. Moreover, deeper insight into biotic interactions within microbial communities is thus crucial to understand adaptation to novel environments. It can help in deciphering the dynamics of living systems and to predict responses to anthropogenic changes in the natural environment (Winder and Schindler, 2004; Davis et al., 2005; Berg et al., 2010).

\section{EE With Natural Microbial Communities in Complex Environments}

Numerous studies have taken an interest in plant-associated microbiota, illustrating the ability of the microorganisms to positively influence plant health and developmental traits such as disease resistance, herbivory, abiotic stress tolerance and growth (Mendes et al., 2011; Grunseich et al., 2020). During plant development, plant-microbiota interactions can change, which may in turn impact plant development (Swenson et al., 2000). For instance, an artificial microcosm selection carried on A. thaliana showed that plant biomass levels can be modified by soil microorganisms. Several $A$. thaliana seeds were inoculated with non-sterile soil batch and let grow for 35 days, which corresponds to one microcosm cycle. After each cycle, the plants presenting the highest and lowest biomasses were selected. The soil from these plants was retrieved and used to inoculate the next batch of plants. The experiment was carried for 16 microcosm cycles and both artificial selections (high or low biomass) were analyzed. Arabidopsis inoculated with the soil community from "high biomass" plants presented indeed higher biomasses than those inoculated with the soil community from "low biomass" plants. After 13 cycles, a soil analysis revealed different soil characteristics for high and low biomasses, that may reflect differences in the biotic components of the corresponding soils (Swenson et al., 2000; Figure 1).

Plant flowering is another trait that can be modulated by plantassociated microorganisms and the contributing microbiota's populations can be experimentally selected and enriched from one microcosm cycle to the next. In a similar way to Swenson and collaborators' experiment, several microcosms of A. thaliana Col0 were created with seeds placed on sterile soil. In this experiment, microcosms were selected for either an early or late flowering phenotype. Plants were harvested and the soil retrieved as soon as all the plants of the microcosm flowered, therefore, the duration of the microcosm cycle depended on the flowering time. After ten cycles on A. thaliana Col0, microorganisms were retrieved and inoculated on other $A$. thaliana genotypes and on a related crucifer, Brassica rapa. Plant-associated microorganisms induced on these plants the same phenotype (early flowering) as the one induced on the genotype (Col0) used for selecting the evolved community (Panke-Buisse et al., 2015). Rather than imposing no selective pressure on the plant host, in these studies, researchers selected a particular plant trait (biomass or flowering) to select plant-associated microorganisms contributing to this plant trait (Table 1). In a similar approach, Lu et al. (2018) selected evolved microbiota capable of either stimulating precocious flowering or delaying it. By studying the molecular interactions between root exudates and the microbiota, a new network of molecular interactions was established, linking the production of auxin phytohormone (i.e., indole-3-acetic acid) from tryptophan by the microbiota, the nitrogen cycling and the timing of flowering in the host plant. EE on natural communities thus enabled to document novel metabolic networks in which soil microbiota influenced plant flowering time, thus shedding light on the key role of soil microbiota on plant functioning.

To better understand the holobiont's mechanistic functioning, an EE study was recently done without taking into consideration improved plant health, growth or development phenotype as a selective pressure outcome of the evolutive process (Morella et al., 2020). The authors collected the leaf microbiota (phyllosphere) of field-grown tomato plants in order to spray it on fresh tomato 
plants. After 10 days of growth, the phyllosphere microbiota were sampled again, and used to inoculate a new round of plants. A total of four passages was done. They compare the impact of five genotypes differing in disease resistance genes in the selection of the tomato's phyllosphere microbiota. They evidenced a strong selection of a stable microbiota adapted to this ecological niche, with a significant driven effect of the tomato genotype. Contrariwise to the initial leaf microbiota that was unstable, the evolved microbiota became well adapted to its host and robust to the invasion of the initial community (Morella et al., 2020).

In the rhizosphere, networks of within-microbiota interactions may also shape the evolution and stability of the community as a whole and the observed effects on the plant, affecting its development, health, and response to abiotic and biotic stresses (Figure 2). Deciphering the principles that underlying ecological and evolutionary properties of microbial communities can allow to build predictive models of ecological dynamics of microbial communities.

\section{CONCLUSION AND FUTURE AVENUES FOR EE STUDIES}

In natural conditions, microbes often coevolve within an interspecific community that may interact with other nonmicrobial organisms, such as plants. Understanding what drives all the different types of interactions with and within microbial communities, and, importantly, how they co-evolved, will allow to draw predictions on how microbial communities and their hosts respond to environmental changes.

EE assays have historically mostly focused on low complexity systems, allowing to understand the evolutionary adaptation of microorganisms to stressful conditions. EE studies combined with whole-genome sequencing have allowed us to understand the genetic bases separating an evolved clone from its original

\section{REFERENCES}

Adams, J., and Rosenzweig, F. (2014). Experimental microbial evolution: history and conceptual underpinnings. Genomics 104, 393-398. doi: 10.1016/j.ygeno. 2014.10.004

Bai, Y., Müller, D. B., Srinivas, G., Garrido-Oter, R., Potthoff, E., Rott, M., et al. (2015). Functional overlap of the Arabidopsis leaf and root microbiota. Nature 528, 364-369. doi: 10.1038/nature 16192

Bailey, S. F., and Bataillon, T. (2016). Can the experimental evolution programme help us elucidate the genetic basis of adaptation in nature? Mol. Ecol. 25, 203-218. doi: $10.1111 / \mathrm{mec} .13378$

Barrett, R. D. H., MacLean, R. C., and Bell, G. (2005). Experimental evolution of Pseudomonas fluorescens in simple and complex environments. Am. Nat. 166, 470-480. doi: 10.1086/444440

Barrick, J. E., Yun, D. S., Yoon, S. H., Jeong, H., Oh, T. K., Schneider, D., et al. (2009). Genome evolution and adaptation in a long-term experiment with Escherichia coli. Nature 461, 1243-1247. doi: 10.1038/nature 08480

Batstone, R. T., Dutton, E. M., Wang, D., Yang, M., and Frederickson, M. E. (2017). The evolution of symbiont preference traits in the model legume Medicago truncatula. New Phytol. 213, 1850-1861. doi: 10.1111/nph. 14308 ancestor strain in a wide range of situations and to reveal the genetic and functional networks involved in microbial adaptation (Segrè et al., 2006; Bailey and Bataillon, 2016; Figure 1). To date, there have been very few EE studies done on more complex systems using natural microbial communities (Table 1). Indeed, whole genome sequencing of many individual clones from one population or from several populations evolving together is a big technological barrier to solve (Figure 1).

The combination of recent technological advances on metaomic approaches, cell sorting and single-cell sequencing, will soon allow to investigate more deeply the genetic mechanisms underlying species-specific adaptation within microbial communities evolving in complex and heterogeneous environments, like the rhizosphere (Box 1 and Table 1). Genetic changes, random genetic drift and natural selection operate on each community member leading to the fixation of mutations, hence altering the genetic composition of populations and indirectly affecting species interactions that dictate community ecology. The intersection of ecology and evolution is key to understand microbial communities.

\section{AUTHOR CONTRIBUTIONS}

$\mathrm{BM}$ wrote the first version of the manuscript and figures. $\mathrm{BM}, \mathrm{DM}$, and $\mathrm{CP}-\mathrm{C}$ corrected and improved the manuscript. All authors have read and agreed to the submitted version of the manuscript.

\section{FUNDING}

BM was supported by a Ph.D. fellowship from the French Ministère de l'Education Nationale, de l'Enseignement Supérieur et de la Recherche. The authors acknowledge funding from the French National Programme EC2CO "Structuring initiative Continental and Coastal Ecosphere"-Microbien 2018-2019.

Batstone, R. T., O’Brien, A. M., Harrison, T. L., and Frederickson, M. E. (2020). Experimental evolution makes microbes more cooperative with their local host genotype. Science 370, 476-478. doi: 10.1126/science.abb7222

Bedhomme, S., Lafforgue, G., and Elena, S. F. (2012). Multihost experimental evolution of a plant RNA virus reveals local adaptation and host-specific mutations. Mol. Biol. Evol. 29, 1481-1492. doi: 10.1093/molbev/msr314

Berg, M. P., Kiers, E. T., Driessen, G., van der Heijden, M., Kooi, B. W., Kuenen, F., et al. (2010). Adapt or disperse: understanding species persistence in a changing world. Glob. Change Biol. 16, 587-598. doi: 10.1111/j.1365-2486.2009.02 014.x

Besset-Manzoni, Y., Rieusset, L., Joly, P., Comte, C., and Prigent-Combaret, C. (2018). Exploiting rhizosphere microbial cooperation for developing sustainable agriculture strategies. Environ. Sci. Pollut. Res. 25, 29953-29970. doi: 10.1007/s11356-017-1152-2

Blount, Z., Barrick, J., Davidson, C., and Lenski, R. E. (2012). Genomic analysis of a key innovation in an experimental Escherichia coli population. Nature 489, 513-518. doi: 10.1038/nature11514

Bouffaud, M. L., Renoud, S., Moënne-Loccoz, Y., and Muller, D. (2016). Is plant evolutionary history impacting recruitment of diazotrophs and nifH expression in the rhizosphere? Sci. Rep. 6:21690. doi: 10.1038/srep21690

Brockhurst, M. A., and Koskella, B. (2013). Experimental coevolution of species interactions. Trends Ecol. Evol. 28, 367-375. doi: 10.1016/j.tree.2013.02.009 
Brockhurst, M. A., Morgan, A. D., Rainey, P. B., and Buckling, A. (2003). Population mixing accelerates coevolution: population mixing accelerates coevolution. Ecol. Lett. 6, 975-979.

Bruto, M., Prigent-Combaret, C., Muller, D., and Moënne-Loccoz, Y. (2014). Analysis of genes contributing to plant-beneficial functions in plant growthpromoting rhizobacteria and related Proteobacteria. Sci. Rep. 4:6261.

Buckling, A., and Rainey, P. B. (2002). Antagonistic coevolution between a bacterium and a bacteriophage. Proc. R. Soc. Lond. B. 269, 931-936. doi: 10. 1098/rspb.2001.1945

Buckling, A., Wills, M. A., and Colegrave, N. (2003). Adaptation limits diversification of experimental bacterial populations. Science 302, 2107-2109. doi: $10.1126 /$ science. 1088848

Burt, A., and Trivers, R. (2009). Genes in Conflict: The Biology of Selfish Genetic Elements. Cambridge, MA: Harvard University Press.

Cairns, J., Jokela, R., Hultman, J., Tamminen, M., Virta, M., and Hiltunen, T. (2018a). Construction and characterization of synthetic bacterial community for experimental ecology and evolution. Front. Genet. 9:312. doi: 10.3389/fgene. 2018.00312

Cairns, J., Ruokolainen, L., Hultman, J., Tamminen, M., Virta, M., and Hiltunen, T. (2018b). Ecology determines how low antibiotic concentration impacts community composition and horizontal transfer of resistance genes. Commun. Biol. 1:35. doi: 10.1038/s42003-018-0041-7

Calcagno, V., Jarne, P., Loreau, M., Mouquet, N., and David, P. (2017). Diversity spurs diversification in ecological communities. Nat. Commun. 8:15810.

Capela, D., Marchetti, M., Clérissi, C., Perrier, A., Guetta, D., Gris, C., et al. (2017). Recruitment of a lineage-specific virulence regulatory pathway promotes intracellular infection by a plant pathogen experimentally evolved into a legume symbiont. Mol. Biol. Evol. 34, 2503-2521. doi: 10.1093/molbev/msx165

Castledine, M., Padfield, D., and Buckling, A. (2020). Experimental (co)evolution in a multi-species microbial community results in local maladaptation. Ecol. Lett. 23, 1673-1681. doi: 10.1111/ele.13599

Chaparro, J. M., Badri, D. V., and Vivanco, J. M. (2014). Rhizosphere microbiome assemblage is affected by plant development. ISME J. 8, 790-803.

Chomicki, G., Kiers, E. T., and Renner, S. S. (2020). The evolution of mutualistic dependence. Annu. Rev. Ecol. Evol. Syst. 51, 409-432.

Cira, N. J., Pearce, M. T., and Quake, S. R. (2018). Neutral and selective dynamics in a synthetic microbial community. Proc. Natl. Acad. Sci. U.S.A. 115, E9842E9848. doi: 10.1073/pnas.1808118115

Colosimo, P. F. (2005). Widespread parallel evolution in sticklebacks by repeated fixation of ectodysplasin alleles. Science 307, 1928-1933. doi: 10.1126/science. 1107239

Consuegra, J., Gaffé, J., Lenski, R. E., Hindré, T., Barrick, J. E., Tenaillon, O., et al. (2021). Insertion-sequence-mediated mutations both promote and constrain evolvability during a long-term experiment with bacteria. Nat. Commun. 12:980. doi: 10.1038/s41467-021-21210-7

Cooper, T. F., Rozen, D. E., and Lenski, R. E. (2003). Parallel changes in gene expression after 20,000 generations of evolution in Escherichia coli. Proc. Natl. Acad. Sci. U.S.A. 100, 1072-1077. doi: 10.1073/pnas.0334340100

Cooper, V. S., and Lenski, R. E. (2000). The population genetics of ecological specialization in evolving Escherichia coli populations. Nature 407, 736-739. doi: $10.1038 / 35037572$

Couce, A., and Tenaillon, O. A. (2015). The rule of declining adaptability in microbial evolution experiments. Front. Genet. 6:99. doi: 10.3389/fgene.2015. 00099

Davis, M. B., Shaw, R. G., and Etterson, J. R. (2005). Evolutionary responses to changing climate. Ecology 86, 1704-1714. doi: 10.1890/03-0788

Derrien, D., Marol, C., and Balesdent, J. (2004). The dynamics of neutral sugars in the rhizosphere of wheat. An approach by $13 \mathrm{C}$ pulse-labelling and GC/C/IRMS. Plant Soil 267, 243-253. doi: 10.1007/s11104-005-5348-8

Doin de Moura, G. G., Remigi, P., Masson-Boivin, C., and Capela, D. (2020). Experimental evolution of legume symbionts: What have we learnt? Genes 11:339. doi: 10.3390/genes11030339

Elena, S. F., and Lenski, R. E. (2003). Microbial genetics: evolution experiments with microorganisms: the dynamics and genetic bases of adaptation. Nat. Rev. Genet. 4, 457-469. doi: 10.1038/nrg1088

Fiegna, F., Moreno-Letelier, A., Bell, T., and Barraclough, T. G. (2015). Evolution of species interactions determines microbial community productivity in new environments. ISME J. 9, 1235-1245. doi: 10.1038/ismej.2014.215
Fisher, R. A. (1930). The Genetical Theory of Natural Selection. Oxford: Oxford University Press.

Flohr, R. C., Blom, C. J., Rainey, P. B., and Beaumont, H. J. (2013). Founder niche constrains evolutionary adaptive radiation. Proc. Natl. Acad. Sci. U.S.A. 110, 20663-20668. doi: 10.1073/pnas.1310310110

Gandon, S., Hochberg, M. E., Holt, R. D., and Day, T. (2013). What limits the evolutionary emergence of pathogens? Philos. Trans. R. Soc. B 368:20120086. doi: 10.1098/rstb.2012.0086

Garoff, L., Pietsch, F., Huseby, D. L., Lilja, T., Brandis, G., and Hughes, D. (2020). Population bottlenecks strongly influence the evolutionary trajectory to fluoroquinolone resistance in Escherichia coli. Mol. Biol. Evol. 37, 1637-1646. doi: $10.1093 / \mathrm{molbev} / \mathrm{msaa} 032$

Genin, S., and Denny, T. P. (2012). Pathogenomics of the Ralstonia solanacearum species complex. Annu. Rev. Phytopat. 50, 67-89. doi: 10.1146/annurev-phyto081211-173000

Gerrish, P. J., and Lenski, R. E. (1998). The fate of competing beneficial mutations in an asexual population. Mutation Evol. 7, 12-144. doi: 10.1007/978-94-0115210-5_12

Gilbert, G. S., and Parker, I. M. (2010). Rapid evolution in a plant-pathogen interaction and the consequences for introduced host species: pathogen evolution and novel hosts. Evol. Appl. 3, 144-156. doi: 10.1111/j.1752-4571. 2009.00107.x

Gopalan-Nair, R., Jardinaud, M. F., Legrand, L., Landry, D., Barlet, X., LopezRoques, C., et al. (2020). Convergent rewiring of the virulence regulatory network promotes adaptation of Ralstonia solanacearum on resistant tomato. Mol; Biol. Evol. doi: 10.1093/molbev/msaa320 [Epub ahead of print].

Gorter, F. A., Manhart, M., and Ackermann, M. (2020). Understanding the evolution of interspecies interactions in microbial communities. Philos. Trans. R. Soc. B. 375:20190256. doi: 10.1098/rstb.2019.0256

Gresham, D., and Hong, J. (2015). The functional basis of adaptive evolution in chemostats. FEMS Microbiol. Rev. 39, 2-16. doi: 10.1111/1574-6976.12082

Grunseich, J. M., Thompson, M. N., Aguirre, N. M., and Helms, A. M. (2020). The role of plant-associated microbes in mediating host-plant selection by insect herbivores. Plants 9:6. doi: 10.3390/plants9010006

Guan, S. H., Gris, C., Cruveiller, S., Pouzet, C., Tasse, L., Leru, A., et al. (2013). Experimental evolution of nodule intracellular infection in legume symbionts. ISME J. 7, 1367-1377. doi: 10.1038/ismej.2013.24

Gude, S., Pinçe, E., Taute, K. M., Seinen, A. B., Shimizu, T. S., and Tans, S. J. (2020). Bacterial coexistence driven by motility and spatial competition. Nature 578 , 588-592. doi: 10.1038/s41586-020-2033-2

Guidot, A., Jiang, W., Ferdy, J.-B., Thébaud, C., Barberis, P., Gouzy, J., et al. (2014). Multihost experimental evolution of the pathogen Ralstonia solanacearum unveils genes involved in adaptation to plants. Mol. Biol. Evol. 31, 2913-2928. doi: 10.1093/molbev/msu229

Hall, A. R., Ashby, B., Bascompte, J., and King, K. C. (2020). Measuring coevolutionary dynamics in species-rich communities. Trends Ecol. Evol. 35, 539-550. doi: 10.1016/j.tree.2020.02.002

Hansen, S. K., Haagensen, J. A., Gjermansen, M., Jørgensen, T. M., Tolker-Nielsen, T., and Molin, S. (2007). Characterization of a Pseudomonas putida rough variant evolved in a mixed-species biofilm with Acinetobacter sp. strain C6. J. Bacteriol. 189, 4932-4943. doi: 10.1128/JB.00041-07

Hartwell, L. H., Hopfield, J. J., Leibler, S., and Murray, A. W. (1999). From molecular to modular cell biology. Nature 402, C47-C52. doi: 10.1038/ 35011540

Herring, C. D., Raghunathan, A., Honisch, C., Patel, T., Applebee, M. K., Joyce, A. R., et al. (2006). Comparative genome sequencing of Escherichia coli allows observation of bacterial evolution on a laboratory timescale. Nat. Genet. 38, 1406-1412. doi: 10.1038/ng1906

Hillesland, K. L., and Stahl, D. A. (2010). Rapid evolution of stability and productivity at the origin of a microbial mutualism. Proc. Natl. Acad. Sci. U.S.A. 107, 2124-2129. doi: 10.1073/pnas.0908456107

Jousset, A., Eisenhauer, N., Merker, M., Mouquet, N., and Scheu, S. (2016). High functional diversity stimulates diversification in experimental microbial communities. Sci. Adv. 2, e1600124. doi: 10.1126/sciadv.160 0124

Jerison, E. R., and Desai, M. M. (2015). Genomic investigations of evolutionary dynamics and epistasis in microbial evolution experiments. Curr. Opin. Genet Dev. 35, 33-39. doi: 10.1016/j.gde.2015.08.008 
Kawecki, T. J., Lenski, R. E., Ebert, D., Hollis, B., Olivieri, I., and Whitlock, M. C. (2012). Experimental evolution. Trends Ecol. Evol. 27, 547-560. doi: 10.1016/j. tree.2012.06.001

Kayser, J., Schreck, C. F., Yu, Q., Gralka, M., and Hallatschek, O. (2018). Emergence of evolutionary driving forces in pattern-forming microbial populations. Philos. Trans. R. Soc. Lond. B. Biol. Sci. 373, 20170106. doi: 10.1098/rstb.2017.0106

Koza, A., Kusmierska, A., McLaughlin, K., Moshynets, O., and Spiers, A. J. (2017). Adaptive radiation of Pseudomonas fluorescens SBW25 in experimental microcosms provides an understanding of the evolutionary ecology and molecular biology of A-L interface biofilm formation. FEMS Microbiol. Lett. 364:fnx109. doi: 10.1093/femsle/fnx109

Kram, K. E., Geiger, C., Ismail, W. M., Lee, H., Tang, H., Foster, P. L., et al. (2017). Adaptation of Escherichia coli to long-term Serial passage in complex medium: evidence of parallel evolution. Systems 2:e0192-16. doi: 10.1128/mSystems. 00192-16

Kuzyakov, Y., and Razavi, B. S. (2019). Rhizosphere size and shape: temporal dynamics and spatial stationarity. Soil Biol. Biochem. 135, 343-360. doi: 10. 1016/j.soilbio.2019.05.011

Lang, G. I., Botstein, D., and Desai, M. M. (2011). Genetic variation and the fate of beneficial mutations in asexual populations. Genetics 188, 647-661. doi: 10.1534/genetics.111.128942

Lawrence, D., Fiegna, F., Behrends, V., Bundy, J. G., Phillimore, A. B., Bell, T., et al. (2012). Species interactions alter evolutionary responses to a novel environment. PLoS Biol. 10:e1001330. doi: 10.1371/journal.pbio.1001330

Lenski, R. E. (2017). Experimental evolution and the dynamics of adaptation and genome evolution in microbial populations. ISME J. 11, 2181-2194. doi: 10.1038/ismej.2017.69

Lenski, R. E., Rose, M. R., Simpson, S. C., and Tadler, S. C. (1991). Long-term experimental evolution in Escherichia coli. I. Adaptation and divergence during 2,000 generations. Am. Nat. 138, 1315-1341. doi: 10.1086/285289

Lenski, R. E., and Travisano, M. (1994). Dynamics of adaptation and diversification: a 10,000-generation experiment with bacterial populations. Proc. Natl. Acad. Sci. U.S.A. 91, 6808-6814. doi: 10.1073/pnas.91.15.6808

Lescat, M., Launay, A., Ghalayini, M., Magnan, M., Glodt, J., Pintard, C., et al. (2017). Using long-term experimental evolution to uncover the patterns and determinants of molecular evolution of an Escherichia coli natural isolate in the streptomycin-treated mouse gut. Mol. Ecol. 26, 1802-1817. doi: 10.1111/mec. 13851

Li, E., Jiang, H., Pieterse, C. M. J., Jousset, A., Bakker, P. A. H. M., and de Jonge, R. (2020). Experimental evolution-driven identification of Arabidopsis rhizosphere competence genes in Pseudomonas protegens. bioRxiv [Preprint]. doi: 10.1101/2020.12.01.407551

Little, A. E., Robinson, C. J., Peterson, S. B., Raffa, K. F., and Handelsman, J. (2008). Rules of engagement: interspecies interactions that regulate microbial communities. Annu. Rev. Microbiol. 62, 375-401. doi: 10.1146/annurev.micro. 030608.101423

Lu, T., Ke, M., Lavoie, M., Jin, Y., Fan, X., and Zhang, Z. (2018). Rhizosphere microorganisms can influence the timing of plant flowering. Microbiome 6:231. doi: 10.1186/s40168-018-0615-0

Maharjan, R., Seeto, S., Notley-McRobb, L., and Ferenci, T. (2006). Clonal adaptive radiation in a constant environment. Science 313, 514-517. doi: 10.1126/science. 1129865

Marchetti, M., Capela, D., Glew, M., Cruveiller, S., Chane-Woon-Ming, B., Gris, C., et al. (2010). Experimental evolution of a plant pathogen into a legume symbiont. PLoS Biol. 8:e1000280. doi: 10.1371/journal.pbio.1000280

Marchetti, M., Clerissi, C., Yousfi, Y., Gris, C., Bouchez, O., Rocha, E., et al. (2017). Experimental evolution of rhizobia may lead to either extra- or intracellular symbiotic adaptation depending on the selection regime. Mol. Ecol. 26, 18181831. doi: 10.1111/mec.13895

Mas, A., Jamshidi, S., Lagadeuc, Y., Eveillard, D., and Vandenkoornhuyse, P. (2016). Beyond the black Queen hypothesis. ISME J. 10, 2085-2091. doi: 10.1038/ismej. 2016.22

McDonald, M. J. (2019). Microbial experimental evolution - a proving ground for evolutionary theory and a tool for discovery. EMBO Rep. 20:e46992. doi: $10.15252 / \mathrm{embr} .201846992$

Meaden, S., and Koskella, B. (2017). Adaptation of the pathogen, Pseudomonas syringae, during experimental evolution on a native vs. alternative host plant. Mol. Ecol. 26, 1790-1801. doi: 10.1111/mec.14060
Mendes, R., Kruijt, M., de Bruijn, I., Dekkers, E., van der Voort, M., Schneider, J. H. M., et al. (2011). Deciphering the rhizosphere microbiome for diseasesuppressive bacteria. Science 332, 1097-1100. doi: 10.1126/science.1203980

Morella, N. M., Weng, F. C., Joubert, P. M., Metcalf, C. J. E., Lindow, S., and Koskella, B. (2020). Successive passaging of a plant-associated microbiome reveals robust habitat and host genotype-dependent selection. Proc. Natl. Acad. Sci. U.S.A. 117, 1148-1159. doi: 10.1073/pnas. 1908600116

Nair, R. R., Vasse, M., Wielgoss, S., Sun, L., Yu, Y. N., and Velicer, G. J. (2019). Bacterial predator-prey coevolution accelerates genome evolution and selects on virulence-associated prey defences. Nat. Commun. 10:4301. doi: 10.1038/ s41467-019-12140-6

Oliveira, N. M., Niehus, R., and Foster, K. R. (2014). Evolutionary limits to cooperation in microbial communities. Proc. Natl. Acad. Sci. U.S.A. 111, 1794117946. doi: 10.1073/pnas.1412673111

Osmond, M. M., and de Mazancourt, C. (2013). How competition affects evolutionary rescue. Philos. Trans. R. Soc. Lond. B 368:20120085. doi: 10.1098/ rstb.2012.0085

Panke-Buisse, K., Poole, A. C., Goodrich, J. K., Ley, R. E., and Kao-Kniffin, J. (2015). Selection on soil microbiomes reveals reproducible impacts on plant function. ISME J. 9, 980-989. doi: 10.1038/ismej.2014.196

Paterson, S., Vogwill, T., Buckling, A., Benmayor, R., Spiers, A. J., Thomson, N. R., et al. (2010). Antagonistic coevolution accelerates molecular evolution. Nature 464, 275-278. doi: 10.1038/nature08798

Perrier, A., Peyraud, R., Rengel, D., Barlet, X., Lucasson, E., Gouzy, J., et al. (2016). Enhanced in planta fitness through adaptive mutations in EfpR, a dual regulator of virulence and metabolic functions in the plant pathogen Ralstonia solanacearum. PLoS Pathog. 12:e1006044. doi: 10.1371/journal.ppat.1006044

Piccardi, P., Vessman, B., and Mitri, S. (2019). Toxicity drives facilitation between 4 bacterial species. Proc. Natl. Acad. Sci. U.S.A. 116, 15979-15984. doi: 10.1073/ pnas. 1906172116

Pickersgill, B. (2018). Parallel vs. convergent evolution in domestication and diversification of crops in the Americas. Front. Ecol. Evol. 6:56. doi: 10.3389/ fevo.2018.00056

Rainey, P. B., and Quistad, S. D. (2020). Toward a dynamical understanding of microbial communities. Philos. Trans. R. Soc. B 375:20190248. doi: 10.1098/rstb. 2019.0248

Ratcliff, W. C., Denison, R. F., Borrello, M., and Travisano, M. (2012). Experimental evolution of multicellularity. Proc. Natl. Acad. Sci. U.S.A. 109, 1595-1600. doi: 10.1073/pnas.1115323109

Ratzke, C., and Gore, J. (2018). Modifying and reacting to the environmental pH can drive bacterial interactions. PLoS Biol. 16:e2004248. doi: 10.1371/journal. pbio. 2004248

Rendueles, O., and Velicer, G. J. (2017). Evolution by flight and fight: diverse mechanisms of adaptation by actively motile microbes. ISME J. 11, 555-568. doi: 10.1038 /ismej.2016.115

Rendueles, O., and Velicer, G. J. (2020). Hidden paths to endless forms most wonderful: complexity of bacterial motility shapes diversification of latent phenotypes. BMC Evol. Biol. 20:145. doi: 10.1186/s12862-020-01707-3

Renoud, S., Bouffaud, M. L., Dubost, A., Prigent-Combaret, C., Legendre, L., Moënne-Loccoz, Y., et al. (2020). Co-occurrence of rhizobacteria with nitrogen fixation and/or 1-aminocyclopropane-1-carboxylate deamination abilities in the maize rhizosphere. FEMS Microbiol. Ecol. 96:fiaa062. doi: 10.1093/femsec/ fiaa062

Rodríguez-Verdugo, A., and Ackermann, M. (2021). Rapid evolution destabilizes species interactions in a fluctuating environment. ISME J. 15, 450-460. doi: 10.1038/s41396-020-00787-9

Sachs, J. L., and Wilcox, T. P. (2006). A shift to parasitism in the jellyfish symbiont Symbiodinium microadriaticum. Proc. R. Soc. B Biol. Sci. 273, 425-429. doi: 10.1098/rspb.2005.3346

Scanlan, P. D., Hall, A. R., Lopez-Pascua, L. D. C., and Buckling, A. (2011). Genetic basis of infectivity evolution in a bacteriophage. Mol. Ecol. 20, 981-989. doi: 10.1111/j.1365-294X.2010.04903.x

Scheuerl, T., Cairns, J., Becks, L., and Hiltunen, T. (2019). Predator coevolution and prey trait variability determine species coexistence. Proc. R. Soc. B 286:20190245. doi: 10.1098/rspb.2019.0245

Scheuerl, T., Hopkins, M., Nowell, R. W., Rivett, D. W., Barraclough, T. G., and Bell, T. (2020). Bacterial adaptation is constrained in complex communities. Nat. Commun. 11:754. doi: 10.1038/s41467-020-14570-z 
Schluter, D., Clifford, E., Nemethy, M., and Mckinnon, J. (2004). Parallel evolution and inheritance of quantitative traits. Am. Nat. 163, 809-822. doi: 10.1086/ 383621

Segrè, A. V., Murray, A. W., and Leu, J.-Y. (2006). High-resolution mutation mapping reveals parallel experimental evolution in yeast. PLoS Biol. 4:e256. doi: 10.1371/journal.pbio.0040256

Seth, E. C., and Taga, M. E. (2014). Nutrient cross-feeding in the microbial world. Front. Microbiol. 5:350. doi: 10.3389/fmicb.2014.00350

Smith, P., and Schuster, M. (2019). Public goods and cheating in microbes. Curr. Biol. 29, R442-R447. doi: 10.1016/j.cub.2019.03.001

Strotz, L. C., Simões, M., Girard, M. G., Breitkreuz, L., Kimmig, J., and Lieberman, B. S. (2018). Getting somewhere with the Red Queen: chasing a biologically modern definition of the hypothesis. Biol. Lett. 14, 20170734. doi: 10.1098/rsbl. 2017.0734

Swenson, W., Wilson, D. S., and Elias, R. (2000). Artificial ecosystem selection. Proc. Natl. Acad. Sci. U.S.A. 97, 9110-9114. doi: 10.1073/pnas.150237597

Tannières, M., Lang, J., Barnier, C., Shykoff, J. A., and Faure, D. (2017). Quorumquenching limits quorum-sensing exploitation by signal-negative invaders. Sci. Rep. 7:40126. doi: 10.1038/srep40126

Tenaillon, O. (2014). The utility of fisher's geometric model in evolutionary genetics. Annu. Rev. Ecol. Evol. Syst. 45, 179-201. doi: 10.1146/annurev-ecolsys120213-091846

Toft, C., and Andersson, S. G. E. (2010). Evolutionary microbial genomics: insights into bacterial host adaptation. Nat. Rev. Genet. 11, 465-475. doi: 10.1038/ nrg2798

Travisano, M., Mongold, J., Bennett, A., and Lenski, R. (1995). Experimental tests of the roles of adaptation, chance, and history in evolution. Science 267, 87-90. doi: 10.1126/science.7809610

Treves, D. S., Manning, S., and Adams, J. (1998). Repeated evolution of an acetatecrossfeeding polymorphism in long-term populations of Escherichia coli. Mol. Biol. Evol. 15, 789-797. doi: 10.1093/oxfordjournals.molbev.a025984

Tso, G. H. W., Reales-Calderon, J. A., Tan, A. S. M., Sem, X., Le, G. T. T., Tan, T. G., et al. (2018). Experimental evolution of a fungal pathogen into a gut symbiont. Science 362, 589-595. doi: 10.1126/science.aat0537

Vacheron, J., Desbrosses, G., Bouffaud, M. L., Touraine, B., Moënne-Loccoz, Y., Muller, D., et al. (2013). Plant growth-promoting rhizobacteria and root system functioning. Front. Plant Sci. 4:356. doi: 10.3389/fpls.2013.00356

Vacheron, J., Desbrosses, G., Renoud, S., Padilla, R., Walker, V., Muller, D., et al. (2018). Differential contribution of plant-beneficial functions from Pseudomonas kilonensis F113 to root system architecture alterations in Arabidopsis thaliana and Zea mays. Mol. Plant Microbe Interact. 31, 212-223. doi: 10.1094/MPMI-07-17-0185-R

Van den Bergh, B., Swings, T., Fauvart, M., and Jan, M. (2018). Experimental design, population dynamics, and diversity in microbial experimental evolution. Microbiol. Mol. Biol. Rev. 82:e00008-18. doi: 10.1128/MMBR.00 008-18

van Elsas, J. D., Turner, S., and Bailey, M. J. (2003). Horizontal gene transfers in the phytosphere. New Phytol. 157, 525-537. doi: 10.1046/j.1469-8137.2003.00697.x

Van Valen, L. (1977). The red queen. Am. Nat. 111, 809-810. doi: 10.1086/283213

Vandenkoornhuyse, P., Quaiser, A., Duhamel, M., Le Van, A., and Dufresne, A. (2015). The importance of the microbiome of the plant holobiont. New Phytol. 206, 1196-1206.
Vidal, M. C., and Segraves, K. A. (2020). Coevolved mutualists experience fluctuating costs and benefits over time. Evolution 75, 219-230. doi: 10.1111/ evo. 14155

Vorholt, J. A., Vogel, C., Carlström, C. I., and Müller, D. B. (2017). Establishing causality: opportunities of synthetic communities for plant microbiome research. Cell Host Microbe 22, 142-155. doi: 10.1016/j.chom.2017.07.004

Westphal, L. L., Lau, J., Negro, Z., Moreno, I. J., Ismail Mohammed, W., Lee, H., et al. (2018). Adaptation of Escherichia coli to long-term batch culture in various rich media. Res. Microbiol. 169, 145-156. doi: 10.1016/j.resmic.2018.01.003

Winder, M., and Schindler, D. E. (2004). Climate change uncouples trophic interactions in an aquatic ecosystem. Ecology 85, 2100-2106. doi: 10.1890/040151

Wiser, M. J., Ribeck, N., and Lenski, R. E. (2013). Long-term dynamics of adaptation in asexual populations. Science 342, 1364-1367. doi: 10.1126/ science. 1243357

Wisniewski-Dyé, F., Borziak, K., Khalsa-Moyers, G., Alexandre, G., Sukharnikov, L. O., Wuichet, K., et al. (2011). Azospirillum genomes reveal transition of bacteria from aquatic to terrestrial environments. PLoS Genet. 7:e1002430. doi: 10.1371/journal.pgen.1002430

Woods, L. C., Gorrell, R. J., Taylor, F., Connallon, T., Kwok, T., and McDonald, M. J. (2020). Horizontal gene transfer potentiates adaptation by reducing selective constraints on the spread of genetic variation. Proc. Natl. Acad. Sci. U.S.A. 117, 26868-26875. doi: 10.1073/pnas.2005331117

Yan, Q., Lopes, L. D., Shaffer, B. T., Kidarsa, T. A., Vining, O., Philmus, B., et al. (2018). Secondary metabolism and interspecific competition affect accumulation of spontaneous mutants in the GacS-GacA regulatory system in Pseudomonas protegens. mBio 9:e01845-17. doi: 10.1128/mBio.01845-17

Yang, L., Jelsbak, L., Marvig, R. L., Damkiaer, S., Workman, C. T., Rau, M. H., et al. (2011). Evolutionary dynamics of bacteria in a human host environment. Proc. Natl. Acad. Sci. U.S.A. 108, 7481-7486. doi: 10.1073/pnas.1018249108

Yeh, P., Tschumi, A. I., and Kishony, R. (2006). Functional classification of drugs by properties of their pairwise interactions. Nat. Genet. 38, 489-494. doi: 10.1038/ ng1755

Yu, Z., Beck, D. A. C., and Chistoserdova, L. (2017). Natural selection in synthetic communities highlights the roles of Methylococcaceae and Methylophilaceae and suggests differential roles for alternative methanol dehydrogenases in methane consumption. Front. Microbiol. 8:2392. doi: 10.3389/fmicb.2017.02392

Zhang, Z., Gosset, G., Barabote, R., Gonzalez, C. S., Cuevas, W. A., and Saier, M. H. (2005). Functional interactions between the carbon and iron utilization regulators, Crp and Fur, in Escherichia coli. J. Bacteriol. 187, 980-990. doi: 10.1128/JB.187.3.980-990.2005

Conflict of Interest: The authors declare that the research was conducted in the absence of any commercial or financial relationships that could be construed as a potential conflict of interest.

Copyright (c) 2021 Manriquez, Muller and Prigent-Combaret. This is an open-access article distributed under the terms of the Creative Commons Attribution License (CC BY). The use, distribution or reproduction in other forums is permitted, provided the original author(s) and the copyright owner(s) are credited and that the original publication in this journal is cited, in accordance with accepted academic practice. No use, distribution or reproduction is permitted which does not comply with these terms. 